Review

\title{
Selective Capture of Transcribed Sequences: A Promising Approach for Investigating Bacterium-Insect Interactions
}

\author{
Ruisheng An and Parwinder S. Grewal * \\ Department of Entomology, Ohio State University, 1680 Madison Ave, Wooster, OH 44691, USA; \\ E-Mail: an.48@osu.edu \\ * Author to whom correspondence should be addressed; E-Mail: grewal.4@osu.edu.
}

Received: 14 December 2011; in revised form: 17 February 2012 / Accepted: 21 February 2012 / Published: 2 March 2012

\begin{abstract}
Bacterial interactions with eukaryotic hosts are complex processes which vary from pathogenic to mutualistic. Identification of bacterial genes differentially expressed in the host, promises to unravel molecular mechanisms driving and maintaining such interactions. Several techniques have been developed in the past 20 years to investigate bacterial gene expression within their hosts. The most commonly used techniques include in-vivo expression technology, signature-tagged mutagenesis, differential fluorescence induction, and cDNA microarrays. However, the limitations of these techniques in analyzing bacterial in-vivo gene expression indicate the need to develop alternative tools. With many advantages over the other methods for analyzing bacterial in-vivo gene expression, selective capture of transcribed sequences (SCOTS) technique has the prospect of becoming an elegant tool for discovery of genes involved in the bacterium-host interaction. Here, we summarize the advances in SCOTS technique, including its current and potential applications in bacterial gene expression studies under a variety of conditions from in-vitro to in-vivo and from mammals to insects.
\end{abstract}

Keywords: bacterium; host; interaction; gene expression; selective capture of transcribed sequences 


\section{Introduction}

The interactions between bacteria and their eukaryotic hosts require the activity of many gene products. Profiles of bacterial genes that are expressed during interaction with the host may provide valuable information. In the past, much of the knowledge came from experiments with bacteria grown in-vitro, and a variety of in-vitro systems were developed to manipulate the host environment using specific culture conditions such as $\mathrm{pH}$, temperature, and iron levels. Although in-vitro assays have been useful, it is obvious that they cannot accurately reproduce all aspects of bacterial interaction with the host. Today, multiple techniques have become available to scan bacterial genes preferentially expressed under a specific growth condition. These techniques are based on determination of either differentially expressed bacterial genes (transcriptional level) or specifically produced bacterial proteins (translational level). The translational-level methods mainly refer to two-dimensional gel electrophoresis (2-D gel) and multidimensional protein identification technology (MUDPIT). The transcriptional-level methods are either mutagenesis based or PCR-hybridization based. Mutagenesis based methods include in-vivo expression technology (IVET), differential fluorescence induction (DFI), signature-tagged mutagenesis (STM), and essential gene test (EGT). PCR-hybridization based methods include differential-display reverse transcription-PCR (DDRT-PCR), cDNA microarray, and selective capture of transcribed sequences (SCOTS). Among these techniques, 2-D gel, MUDPIT, EGT and DDRT-PCR have been applied for profiling bacterial genes expressed under in-vitro conditions, but not in-vivo. While the rest of the techniques have been originally developed to study the bacterial gene expression in mammalian models, some of them have also been subsequently applied in bacteriainsect interactions. Here, we first briefly discuss most techniques facilitating identification of differentially expressed bacterial genes or gene products in-vitro or in-vivo, and then primarily focus on the recent advances in the SCOTS technique which has many advantages over the other techniques. The main purpose of this paper is to share updated information about the techniques and to describe the proper conditions for the use of each technique particularly the SCOTS technique.

\section{In-vitro Profiling Techniques}

As mentioned above, in-vitro profiling techniques refer to the methods currently being used only for profiling bacterial genes expressed in-vitro but with the potential to be adapted for in-vivo use. Of these techniques, 2-D gel and MUDPIT are translational-level methods, EGT is a mutagenesis based method, and DDRT-PCR is a PCR-hybridization based method. The 2-D gel and MUDPIT have been developed to analyze bacterial proteins differentially produced in a specific growth condition. The 2-D gel relies on the separation of whole proteins by gel electrophoresis from two dimensions and the subsequent identification of individual proteins through mass spectrometry, and the MUDPIT relies on the separation of proteolytic peptides by liquid chromatography and identification by electrospray ionization-tandem mass spectrometry. Comparatively, 2-D gel often excludes large and hydrophobic proteins, and the MUDPIT approach overcomes this limitation; but 2-D gel provides a visual reference to compare protein expression, posttranslational modifications and protein cleavage events, which would not be detected by using MUDPIT. Also, unlike 2-D gel, MUDPIT does not yield quantitative information [1,2]. Thus, in order to overcome the problems limiting the coverage of proteomic 
analysis, both techniques should be complementarily used [2,3]. Theoretically, through comparing protein complexes present in-vitro and in-vivo, 2-D gel can be used to identify in-vivo induced gene products. However, although studies have been reported to profile bacterial proteins during growth in-vitro under conditions that mimic some aspects of infection, up to now, no studies have been published to report the global protein expression of a bacterial species within its natural host or an animal model. This is mainly because of the technical hurdles associated with separating bacteria from the host tissues and obtaining enough material to perform statistical analyses such as quantifying individual proteins and determining their sequence information. While the potential of this technique for bacterial in-vivo gene expression analysis is limited, an improved strategy would be prefractionation, including sequential extractions in increasingly stronger solubilization cocktails, subcellular fractionation, and selective removal of dominant protein components [4] (for review see [5]). Yet, there is still no report on the influence of pre-fractionation to the protein separation quality and quantity.

The essential gene test (EGT) consists of a variety of techniques introduced to identify essential genes that are required for bacterial growth in-vitro or in-vivo. Because such genes are expressed under all conditions, they will not be identified by other methods such as 2-D gel, IVET or STM unless they were expressed at extremely low levels in-vitro relative to in-vivo (for review see [6]). One of the variations for EGT is known as genomic analysis and mapping by in vitro transposition [7-10]. Basically, the EGT strategy includes two steps: the first step involves efficient in vitro transposition mutagenesis and recombination onto the bacterial chromosome, and the second step maps the genomic location of each transposon insertion in a pool of mutants by either genetic footprinting or phenotypic characterization. Like STM, the use of EGT constitutes a negative selection in which certain mutants are eliminated. Theoretically, any bacterial mutants can be recognized by the loss of PCR products [11] or the defect of certain phenotypes [7] in a specific growth condition but presence of these properties in in-vitro broth. The major limitation for EGT is that it can only be applied to naturally competent bacterial cells. The development of efficient DNA transformation methods should enable the adaptation of this strategy for the analysis of bacteria that are not naturally competent [11].

Differential-display reverse transcription-PCR (DDRT-PCR) technique allows analysis of gene expression among several cell populations [12]. It involves reverse transcription of mRNAs isolated from different cell populations with random primers to generate cDNA pools and PCR amplification of cDNA pools. The amplified products are separated and compared by SDS-PAGE gels. DDRT-PCR has been mainly used to compare gene expression between virulent and non-virulent bacterial strains [13,14] (for review see [15]). This technique may provide an unbiased method to compare mRNA pools from two or more samples, but several limitations including difficulty to obtain good quality mRNA and isolation of PCR products, the large number of false-positive results, and difficulty to confirm differential expression, limit its advance in bacterial gene expression studies [15]. 


\section{Approaches in Bacteria-Host Interactions}

\subsection{Mutagenesis Based Methods}

In vivo expression technology (IVET) is a promoter-trap strategy in which a random DNA is linked to a reporter gene required for bacterial growth in the host [16]. This system rests on positive selection to identify genes turned on during in-vivo growth. There are several variations of IVET, and each relies on the generation of transcriptional fusions of genomic sequences to a reporter gene such as purA or antibiotic gene (for review see [17]). One of the limitations in the use of this technique is the requirement of well-developed genetic manipulation systems such as ability to choose suitable reporter genes and to successfully transform and mutagenize the bacteria [17].

Differential fluorescence induction (DFI) is another promoter-trap based method [18]. The differentially expressed genes are identified by a genetic selection via fluorescence-enhanced green fluorescent protein (GFP) and a fluorescence activated cell sorter (FACS). The bacteria are separated by FACS in response to the expression, or lack of expression along with the fluorescent marker. One good example of the application of this approach in bacteria-insect interaction is the identification of genes up-regulated by the bacterium Photorhabdus luminescens after infection of the insect host Galleria mellonella [19]. Using the mCherry fluorophore as a reporter, approximately 13,000 clones were screened for fluorescence induction in the presence of G. mellonella larvae homogenate. Out of these clones, 24 promoters were verified to be induced in viable G. mellonella larvae, and some of them have been already known to regulate the expression of toxin and genes [19]. Similar to IVET, DFI strategy also requires well-developed genetic manipulation systems including ability to select a suitable house-keeping promoter to construct the mutagenesis library and genetic transfer mechanisms.

Signature-tagged mutagenesis (STM) is a negative selection technique in which a pool of sequence-tagged mutant bacteria is administered to the host in an appropriate model [20]. Mutations represented in the initial inoculum but not recovered from the host are identified as essential factors for survival within the host (for review see [21]). However, this technique is limited by the need for an adequate model that facilitates recovery of bacteria from an infected host. Mutants that are slow-growing, not viable, contain mutations in genes encoding redundant functions, or that can be complemented in a mixed population may be unidentified (for review see [21-25]).

\subsection{Hybridization Based Methods}

cDNA microarrays are used to determine the difference in mRNA levels among bacterial strains grown in-vitro and in-vivo [26-30]. A separate dye for each of the two cDNA pools being compared is incorporated into the reverse transcription step. After allowing for hybridization of the cDNA to the microarray, the differential fluorescence of the two cDNA pools is measured and the ratio is determined for each gene on the microarray to reflect the difference in mRNA level. Theoretically, cDNA microarrays offer the promise of accurate gene expression measurements for every gene in a genome. However, this potential has not been realized because of the substantial technical problems. The main problems include the amount of bacterial mRNA needed for the array and the difficulty in purifying bacterial mRNA from the host RNA. Therefore, cDNA microarray can currently only be applied to bacteria with high titers in host tissues [27]. To overcome this limitation, efforts have been 
underway to develop methods requiring less bacterial mRNA, and one example is differential expression analysis by using a custom-amplified library (DECAL). DECAL is used to selectively amplify specific bacterial transcriptomes, and its conjunction with expression microarray can be performed with as little as $10 \mathrm{ng}$ of bacterial mRNA and can detect as low as 4-fold differences in gene expression [31-33]. However, the potential use of DECAL suffers from having limited range for detection of mRNA and cannot provide direct differential quantitation (for review see [34]).

\section{Selective Capture of Transcribed Sequences}

The development of elegant techniques as described above in recent years to study bacterial in-vivo gene expression enables in-depth exploration of bacteria-host interactions. However, there are common limitations for these techniques including the isolation of abundant high quality mRNA and the separation of bacterial cDNA from host cDNA. An improved method, selective capture of transcribed sequences (SCOTS) overcomes these limitations. SCOTS is a PCR-hybridization based approach by which bacterial genes preferentially expressed under a specific growth condition are captured by subtractive hybridization and PCR amplification with the tagged primers (Figure 1). Briefly, total RNA samples isolated from bacteria grown in-vitro or in the host are converted to cDNAs using primers with random sequence at 3'-end and tagged sequence at 5'-end. The synthesized bacterial cDNAs are normalized by hybridization to biotinylated-sonicated bacterial genomic DNA (gDNA) that has been blocked beforehand using bacterial ribosomal RNA (rRNA) sequences. Blocking with rRNA allows an effective capture of cDNA molecules representing mRNA transcripts. The cDNAs-gDNA-rRNA hybrids are captured by streptavidin-coated magnetic beads, eluted, and PCR amplified using the tagged sequence as primers. The amplified cDNAs are denatured, and again hybridized to gDNArRNA mixture for additional rounds of normalization. The normalization process finally results in sampling of bacterial mRNA transcripts apart from its ribosomal and host transcripts. After a subtractive hybridization between normalized in-vitro and in-vivo cDNAs, bacterial cDNAs preferentially upregulated or down-regulated in the host are enriched. The enriched bacterial cDNAs are then cloned into a cloning vector to construct libraries representing bacterial genes up- or down-regulated in the host, and individual clones are screened by southern blot hybridization with probes made from normalized in-vitro or in-vivo cDNAs for further confirmation.

SCOTS approach was originally developed by Graham and Clark-Curtiss [35] for identification of genes expressed by Mycobacterium tuberculosis in macrophages. Since then, SCOTS has been successfully used to profile gene expression in a diversity of bacteria. In-vitro work with Listeria monocytogenes, a food-borne bacterial pathogen able to grow at refrigeration temperatures, has identified 24 different cDNA clones which were involved in cold-adaptive responses, regulatory adaptive responses, general microbial stress responses, amino acid metabolism, cell surface alterations, and degradative metabolism [36]. The gene expression of Helicobacter pylori in the context of persistent infection remained largely uncharacterized before the application of SCOTS [37]. The majority of SCOTS identified cDNAs are corresponding to the factors unique to $H$. pylori that are potentially produced in response to interactions with mammalian gastric mucosa. The pathogenic Escherichia coli strains cause a variety of diseases in different host species, and pathogen-specific genes corresponding to the virulence factors such as adhesins and lipopolysaccharide have been identified from $E$. coli strain 
7122 during infection of chicken by using SCOTS approach [38]. Actinobacillus pleuropneumoniae is the porcine respiratory tract pathogen that presents a major problem to the swine industry due to its ability to persist in the host, surviving in tonsils as well as in sequestered necrotic lung tissue, which leads to the occurrence of subclinically infected carrier animals. Genes expressed by A. pleuropneumoniae in necrotic porcine lung tissue have been identified by SCOTS [39]. A recent study has further demonstrated the feasibility of SCOTS for elucidating bacterial gene expression during pathogenic interaction with the insect host. Using SCOTS technique, a total of 40 genes in the bacterium Photorhabdus temperata and 39 in Xenorhabdus koppenhoeferi have been identified to be up-regulated in the hemolymph of white grub Rhizotrogus majalis [40]. These identified genes are divided into seven functional groups including cell surface structure, regulation, virulence and secretion, stress response, intracellular metabolism, nutrient scavenging, and unknown. More than $60 \%$ of the identified genes were uniquely induced in either bacterium suggesting vastly different molecular mechanisms of pathogenicity to the same insect host. Further analyses of these identified genes have indicated possible mechanistic associations between the identified gene products in metabolic pathways, providing an interactive model of pathogenesis for each bacterium species. Success of SCOTS in other bacterial pathogenic interactions includes Moraxella osloensis in slugs [41], Streptococcus suis in pigs [42], Ehrlichia ruminantium in ruminants [43], Riemerella anatipestifer in ducks [44], and Haemophilus parasuis in necrotic porcine [45], etc.

In situations where the bacterial infection process is unpredictable, it is hard to decide when to terminate the experiment for nucleic acid isolation. Given this requirement, SCOTS derived cDNA probes from the specific growth condition such as broth cultures can be used to screen the bacterial gene expression at the different time points post-infection to monitor the infection process [46]. For example, analysis of the $\operatorname{trc} R$ and $\operatorname{trcS}$ genes using various SCOTS probes confirmed that these genes are expressed in broth-grown cultures and after $18 \mathrm{~h}$ of $M$. tuberculosis growth in cultured human primary macrophages [47]. A related study on the gene Rv1057 by using SCOTS derived probes has shown that this gene is expressed during early M. tuberculosis growth in human macrophages, and its expression correlates to a gene that is repressed by TrcR. Besides being used in a stand-alone fashion, results generated with SCOTS have been used in conjunction with cDNA microarray to validate the identified cDNAs and to determine global gene expression [37,48]. Faucher et al. [48] used this method to study global expression profiles of Salmonella enterica serovar Typhi grown in broth and within macrophages at different time points. The SCOTS-cDNA mixture displayed an expected expression profile of Typhi virulence genes from infected macrophages, and the global expression analysis has identified many hypothetical and characterized Typhi genes that may contribute to adaptation and survival within macrophages.

In addition to profiling bacterial virulence genes, new results have suggested that SCOTS can be used to dissect the symbiotic interactions between bacteria and their host. Luminescent bacteria are well known as colonizers in light organs of both sepiolid and loliginid squids. By examining bacterial transcripts solely expressed in either the light organ or free-living stages, differential gene expression in bacterial symbionts from loliginid squids demonstrates variation between mutualistic and environmental niches. The identified genes specific for squid light organs include vulnibactin synthetase, outer membrane protein $\mathrm{W}$ and dihydroxy dehydratase [49]. Another study in an emerging 
mutualistic model system, insect-pathogenic bacteria Photorhabdus and their insect-parasitic nematode partner Heterorhabditis, based on SCOTS approach, has found that the bacteria undergo major transcriptional reshaping in the nematode intestine. The bacteria induce cellular acidification to slow down growth, switch to pentose phosphate pathway to overcome oxidative stress and nutrition limitation, and shed motility but develop biofilm to persist in the nematode intestine until being released into the insect hemolymph [50].

Finally, SCOTS technique has the potential to determine the differences in gene expression in host cells or tissues by comparative hybridization between different strains that belong to the same or similar species with high overall DNA homology but with different degrees of host specificities [38]. Identification of pathogen-specific and conserved bacterial genes that are expressed in-vivo can provide further insight into the mechanisms by which bacteria colonize host tissues, cope with, or circumvent host defenses and adjust to the nutrient limitations and other stresses that occur in different host environments.

Figure 1. Schematic presentation of the selective capture of transcribed sequences (SCOTS) approach. In panel A, bacterial cDNAs expressed in response to different growth conditions are normalized to separate from the ribosomal and host cDNAs. In panel B, bacterial cDNAs corresponding to genes preferentially up-regulated or down-regulated in the host relative to the broth were enriched by subtractive hybridization (Adapted from An et al. [40] and An and Grewal [50]).

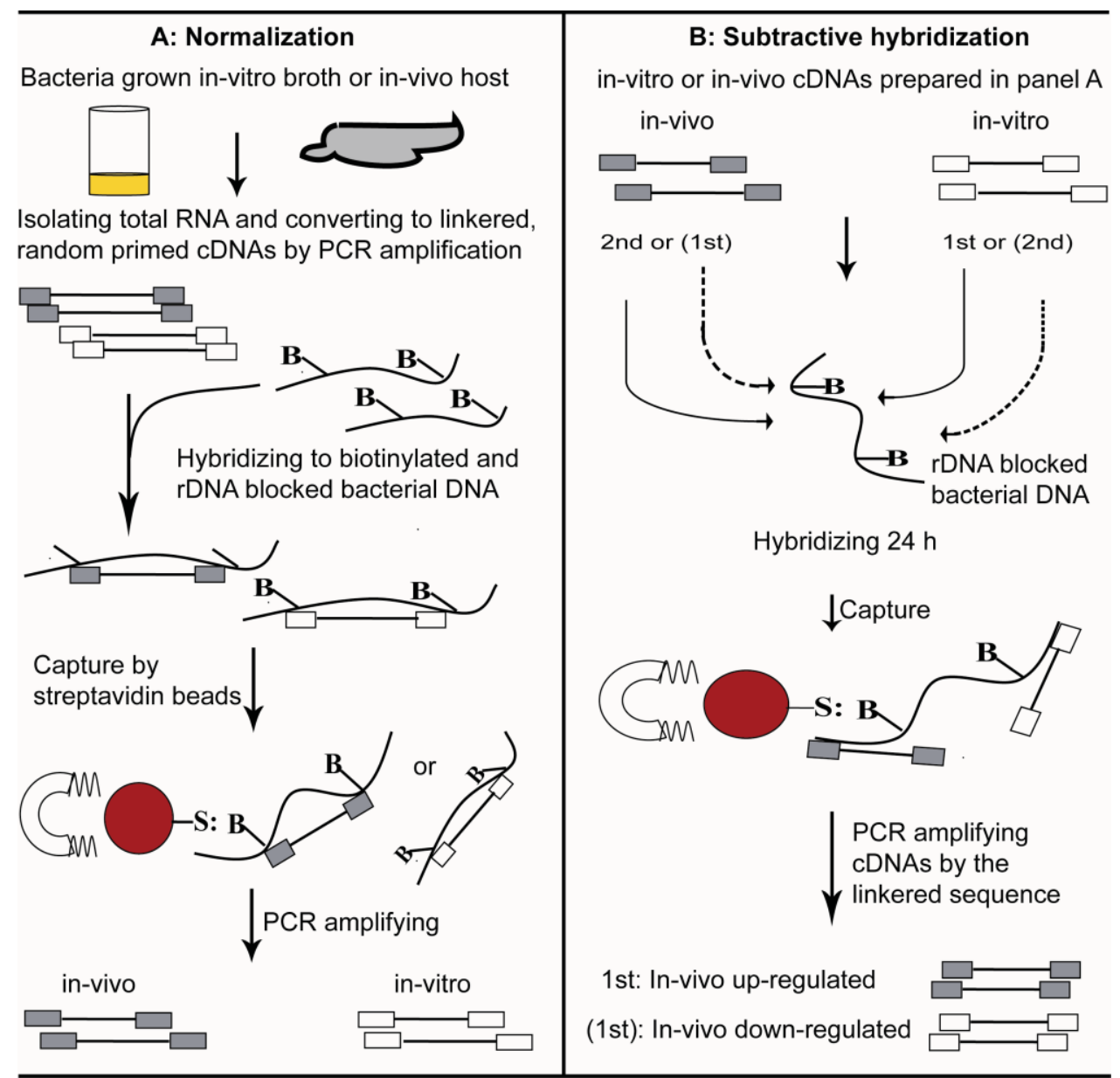




\section{SCOTS versus other Methods}

Theoretically, SCOTS can be applied to any bacterial species, with no requirement for specialized genetic techniques and species-specific cloning vectors (Table 1). Unlike IVET or DFI strategy, SCOTS identifies relevant genes, rather than promoter regions. SCOTS also has the advantage to detect in-vivo gene expression from small numbers of bacterial cells obtained in samples from host tissues in natural states, an application for which other methods are currently not available. Because of this advantage, the combination of SCOTS and cDNA microarrays will be an effective way to determine the bacterial gene expression without the need to recover many nanograms of bacterial mRNA from host and without increasing the bacterial load beyond what is seen in nature. However, the SCOTS technique does have some limitations. For example, it is not a quantitative method for gene expression studies, and the essential genes are not detected. In addition, a common feature for all of the above techniques is that they all need post-process to evaluate and validate the identified genes to be involved in bacteria-host interactions.

Table 1. Comparison of requirements of different approaches in profiling bacterial gene expression.

\begin{tabular}{lllll}
\hline Approach & $\begin{array}{l}\text { Bacteria } \\
\text { Requirements }\end{array}$ & $\begin{array}{l}\text { Animal } \\
\text { Model }\end{array}$ & mRNA Requirements & $\begin{array}{l}\text { Genetic } \\
\text { Information }\end{array}$ \\
\hline 2-D Gel & High titer & Not needed & Not needed & Not needed \\
DDRT-PCR & High titer & Not needed & High quality \& quantity & Needed \\
Microarray & High titer & Not needed & High quality \& quantity & Needed \\
IVET & Transformable & Needed & Not needed & Not needed \\
DFI & Transformable & Needed & Not needed & Not needed \\
STM & Transformable & Needed & Not needed & Not needed \\
GAMBIT & Transformable & Needed & Not needed & Needed \\
SCOTS & No restriction & Not needed & No restriction & Not needed \\
\hline
\end{tabular}

\section{Conclusions}

New methods have been developed to simplify the analysis of bacterial genes actively expressed in the host, and such studies will transform our understanding of the molecular mechanisms driving and maintaining the bacteria-host interactions. It must be emphasized that most of these techniques only identify putative differentially expressed genes and further confirmation on the role of the genes in the bacterial interaction with the host is still necessary. Also, none of the currently available techniques is perfect, and the best strategy usually is to use a combination of techniques. For example, as noted above, while SCOTS can scan bacterial gene expression without need of prior genetic information, its combination with cDNA microarrays makes the data more reliable and quantitative. Therefore, continued improvements in the techniques for acquiring genetic information regarding bacterial in-vivo gene expression is still needed, and the advances will likely be gradual improvements in current technologies rather than new technologies. 


\section{References}

1. Gygi, S.P.; Rist, B.; Gerber, S.A.; Turecek, F.; Gelb, M.H.; Aebersold, R. Quantitative analysis of complex protein mixtures using isotopecoded affinity tags. Nat. Biotechnol. 1999, 17, 994-999.

2. Boyce, J.D.; Cullen, P.A.; Adler, B. Genomic-scale analysis of bacterial gene and protein expression in the host. Emerg. Infect. Dis. 2004, 10, 1357-1362.

3. Schmidt, F.; Donahoe, S.; Hagens, K.; Mattow, J.; Schaible, U.; Kaufmann, S.; Aebersold, R.; Jungblut, P.R. Complementary analysis of the Mycobacterium tuberculosis proteome by two-dimensional electrophoresis and isotope coded affinity tag technology. Mol. Cell Proteomics 2004, 3, 24-42.

4. Zuo, X.; Echan, L.; Hembach, P.; Tang, H.Y.; Speicher, K.D.; Santoli, D.; Speicher, D.W. Towards global analysis of mammalian proteomes using sample prefractionation prior to narrow $\mathrm{pH}$ range two-dimensional gels and using one-dimensional gels for insoluble and large proteins. Electrophoresis 2001, 22, 1603-1615.

5. Hamdan, M.; Righetti, P.G. Assessment of protein expression by means of 2-D gel electrophoresis with and without mass spectrometry. Mass Spectrom. Rev. 2003, 22, 272-284.

6. Lehoux, D.E.; Sanschagrin, F.; Levesque, R.C. Discovering essential and infection-related genes. Curr. Opin. Microbiol. 2001, 4, 515-519.

7. Judson, N.; Mekalanos, J.J. TnAraOut, a transposon-based approach to identify and characterize essential bacterial genes. Nat. Biotechnol. 2000, 18, 740-745.

8. Reich, K.A.; Chovan, L.; Hessler, P. Genome scanning in Haemophilus influenzae for identification of essential genes. J. Bacteriol. 1999, 181, 4961-4968.

9. Wong, S.M.; Mekalanos, J.J. Genetic footprinting with mariner-based transposition in Pseudomonas aeruginosa. Proc. Natl. Acad. Sci. USA 2000, 97, 10191-10196.

10. Hare, R.S.; Walker, S.S.; Dorman, T.E.; Greene, J.R.; Guzman, L.M.; Kenney, T.J.; Sulavik, M.C.; Baradaran, K.; Houseweart, C.; Yu, H.; et al. Genetic footprinting in bacteria. J. Bacteriol. 2001, 183, 1694-1706.

11. Akerley, B.J.; Rubin, E.J.; Lampe, D.J.; Mekalanos, J.J. PCR-mediated detection of growthattenuated mutants in large pools generated by in vitro transposon mutagenesis. Presented at the $98^{\text {th }}$ General Meeting of the American Society for Microbiology, Atlanta, GA, USA, 17-21 May 1998; American Society for Microbiology: Atlanta, GA, USA, 1998.

12. Liang, P.; Pardee, A.B. Differential display of eukaryotic messenger RNA by means of the polymerase chain reaction. Science 1992, 257, 967-971.

13. Rindi, L.; Lari, N.; Garzelli, C. Search for genes potentially involved in Mycobacterium tuberculosis virulence by mRNA differential display. Biochem. Biophys. Res. Commun. 1999, 258, 94-101.

14. Rivera-Marrero, C.A.; Burroughs, M.A.; Masse, R.A.; Vannberg, F.O.; Leimbach, D.L.; Roman, J.; Murtagh, J.J., Jr. Identification of genes differentially expressed in Mycobacterium tuberculosis by differential display PCR. Microb. Pathog. 1998, 25, 307-316.

15. Sturtevant, J. Applications of differential-display reverse transcription-PCR to molecular pathogenesis and medical mycology. Clin. Microbiol. Rev. 2000, 13, 408-427. 
16. Mahan, M.J.; Slauch, J.M.; Mekalanos, J.J. Selection of bacterial virulence genes that are specifically induced in host tissues. Science 1993, 259, 686-688.

17. Angelichio, M.J.; Camilli, A. In vivo expression technology. Infect. Immun. 2002, 70, 6518-6523.

18. Valdivia, R.H.; Falkow, S. Bacterial genetics by flow cytometry: Rapid isolation of Salmonella typhimurium acid-inducible promoters by differential fluorescence induction. Mol. Microbiol. 1996, 22, 367-378.

19. Münch, A.; Stingl, L.; Jung, K.; Heermann, R. Photorhabdus luminescens genes induced upon insect infection. BMC Genomics 2008, 9, 229.

20. Hensel, M.; Shea, J.E.; Gleeson, C.; Jones, M.D.; Dalton, E.; Holden, D.W. Simultaneous identification of bacterial virulence genes by negative selection. Science 1995, 269, 400-403.

21. Mecsas, J. Use of signature-tagged mutagenesis in pathogenesis studies. Curr. Opin. Microbiol. 2002, 5, 33-37.

22. Lehoux, D.E.; Levesque, R.C. Detection of genes essential in specific niches by signature-tagged mutagenesis. Curr. Opin. Biotechnol. 2000, 11, 434-439.

23. Shea, J.E.; Santangelo, J.D.; Feldman, R.G. Signature-tagged mutagenesis in the identification of virulence genes in pathogens. Curr. Opin. Microbiol. 2000, 3, 451-458.

24. Autret, N.; Charbit, A. Lessons from signature-tagged mutagenesis on the infectious mechanisms of pathogenic bacteria. FEMS Microbiol. Rev. 2005, 29, 703-717.

25. Saenz, H.L.; Dehio, C. Signature-tagged mutagenesis: Technical advances in a negative selection method for virulence gene identification. Curr. Opin. Microbiol. 2005, 8, 612-619.

26. Boyce, J.D.; Wilkie, I.; Harper, M.; Paustian, M.L.; Kapur, V.; Adler, B. Genomic-scale analysis of Pasteurella multocida gene expression during growth within liver tissue of chickens with fowl cholera. Microbes Infect. 2004, 6, 290-298.

27. Boyce, J.D.; Wilkie, I.; Harper, M.; Paustian, M.L.; Kapur, V.; Adler, B. Genomic scale analysis of Pasteurella multocida gene expression during growth within the natural chicken host. Infect. Immun. 2002, 70, 6871-6879.

28. Xu, Q.; Dziejman, M.; Mekalanos, J.J. Determination of the transcriptome of Vibrio cholerae during intraintestinal growth and midexponential phase in vitro. Proc. Natl. Acad. Sci. USA 2003, 100, 1286-1291.

29. Paustian, M.L.; May, B.J.; Kapur, V. Pasteurella multocida gene expression in response to iron limitation. Infect. Immun. 2001, 69, 4109-4115.

30. Schembri, M.A.; Kjaergaard, K.; Klemm, P. Global gene expression in Escherichia coli biofilms. Mol. Microbiol. 2003, 48, 253-267.

31. Alland, D.; Kramnik, I.; Weisbrod, T.R.; Otsubo, L.; Cerny, R.; Miller, L.P.; Jacobs, W.R., Jr.; Bloom, B.R. Identification of differentially expressed mRNA in prokaryotic organisms by customized amplification libraries (DECAL): The effect of isoniazid on gene expression in Mycobacterium tuberculosis. Proc. Natl. Acad. Sci. USA 1998, 95, 13227-13232.

32. Narasimhan, S.; Santiago, F.; Koski, R.A.; Brei, B.; Anderson, J.F.; Fish, D.; Fikrig, E. Examinatin of the Borrelia burgdorferi transcriptome in lxodes scapularis during feeding. $J$. Bacteriol. 2002, 184, 3122-3125. 
33. Narasimhan, S.; Camaino, M.J.; Liang, F.T.; Santiago, F.; Laskowski, M.; Philipp, M.T.; Pachner, A.R.; Radolf, J.D.; Fikrig, E. Borrelia burgdorferi transcriptome in the central nervous system of non-human primates. Proc. Natl. Acad. Sci. USA 2003, 100, 15953-15958.

34. Shelburne, S.A.; Musser, J.M. Virulence gene expression in vivo. Curr. Opin. Microbiol. 2004, 7, 283-289.

35. Graham, J.E.; Clark-Curtiss, J.E. Identification of Mycobacterium tuberculosis RNAs synthesized in response to phagocytosis by human macrophages by selective capture of transcribed sequences (SCOTS). Proc. Natl. Acad. Sci. USA 1999, 96, 11554-11559.

36. Liu, S.; Graham, J.E.; Bigelow, L.; Morse, P.D., 2nd; Wilkinson, B.J. Identification of Listeria monocytogenes genes expressed in response to growth at low temperature. Appl. Environ. Microbiol. 2002, 68, 1697-705.

37. Graham, J.E.; Peek, R.M., Jr.; Krishna, U.; Cover, T.L. Global analysis of Helicobacter pylori gene expression in human gastric mucosa. Gastroenterology 2002, 123, 1637-1648.

38. Dozois, C.M.; Daigle, F.; Curtiss, R., 3rd. Identification of pathogen-specific and conserved genes expressed in vivo by an avian pathogenic Escherichia coli strain. Proc. Natl. Acad. Sci. USA 2003, 100, 247-252.

39. Baltes, N.; Gerlach, G.F. Identification of genes transcribed by Actinobacillus pleuropneumoniae in necrotic porcine lung tissue by using selective capture of transcribed sequences. Infect. Immun. 2004, 72, 6711-6716.

40. An, R.; Sreevatsan, S.; Grewal, P.S. Comparative in vivo gene expression of the closely related bacteria Photorhabdus temperata and Xenorhabdus koppenhoeferi upon infection of the same insect host, Rhizotrogus majalis. BMC Genomics 2009, 10, 433.

41. An, R.; Sreevatsan, S.; Grewal, P.S. Moraxella osloensis gene expression in the slug host Deroceras reticulatum. BMC Microbiology 2008, 8, 19.

42. Li, W.; Liu, L.; Qiu, D.; Chen, H.; Zhou, R. Identification of Streptococcus suis serotype 2 genes preferentially expressed in the natural host. Int. J. Med. Microbiol. 2010, 300, 482-488.

43. Emboule, L.; Daigle, F.; Meyer, D.F.; Mari, B.; Pinarello, V.; Sheikboudou, C.; Magnone, V.; Frutos, R.; Viari, A.; Barbry, P.; et al. Innovative approach for transcriptomic analysis of obligate intracellular pathogen: Selective capture of transcribed sequences of Ehrlichia ruminantium. BMC Mol. Biol. 2009, 10, 111.

44. Zhou, Z.; Zheng, J.; Tian, W.; Li, J.; Zhang, W.; Zhang, J.; Meng, X.; Hu, S.; Bi, D.; Li, Z. Identification of Riemerella anatipestifer genes differentially expressed in infected duck livers by the selective capture of transcribed sequences technique. Avian Pathol. 2009, 38, 321-329.

45. Jin, H.; Wan, Y.; Zhou, R.; Li, L.; Luo, R.; Zhang, S.; Hu, J.; Langford, P.R.; Chen, H. Identification of genes transcribed by Haemophilus parasuis in necrotic porcine lung through the selective capture of transcribed sequences (SCOTS). Environ. Microbiol. 2008, 10, 3326-3336.

46. Daigle, F.; Hou, J.Y.; Clark-Curtiss, J.E. Microbial gene expression elucidated by selective capture of transcribed sequences (SCOTS). Methods Enzymol. 2002, 358, 108-122.

47. Haydel, S.E.; Benjamin, W.H., Jr.; Dunlap, N.E.; Clark-Curtiss, J.E. Expression, autoregulation, and DNA binding properties of the Mycobacterium tuberculosis TrcR response regulator. J. Bacteriol. 2002, 184, 2192-2203. 
48. Faucher, S.P.; Porwollik, S.; Dozois, C.M.; McClelland, M.; Daigle, F. Transcriptome of Salmonella enterica serovar Typhi within macrophages revealed through the selective capture of transcribed sequences. Proc. Natl. Acad. Sci. USA 2006, 103, 1906-1911.

49. Guerrero-Ferreira, R.C.; Nishiguchi, M.K. Differential gene expression in bacterial symbionts from loliginid squids demonstrates variation between mutualistic and environmental niches. Environ. Microbiol. Rep. 2010, 2, 514-523.

50. An, R.; Grewal, P.S. Molecular mechanisms of persistence of mutualistic bacteria Photorhabdus in the entomopathogenic nematode host. PLoS One 2010, 5, e13154.

(C) 2012 by the authors; licensee MDPI, Basel, Switzerland. This article is an open access article distributed under the terms and conditions of the Creative Commons Attribution license (http://creativecommons.org/licenses/by/3.0/). 